\title{
Effect of glibenclamide on the prevention of secondary brain injury following ischemic stroke in humans
}

\author{
Arjun Khanna, B.S., ${ }^{1,2}$ Brian P. Walcott, M.D., ${ }^{1,2}$ Kristopher T. Kahle, M.D., Ph.D., ${ }^{1,2}$ \\ and J. Marc Simard, M.D., Ph.D. ${ }^{3}$ \\ ${ }^{1}$ Harvard Medical School; ${ }^{2}$ Department of Neurosurgery, Massachusetts General Hospital, Boston, \\ Massachusetts; and ${ }^{3}$ Departments of Neurosurgery, Pathology, and Physiology, University of Maryland \\ School of Medicine, Baltimore, Maryland
}

\begin{abstract}
Cerebral edema and hemorrhagic conversion are common, potentially devastating complications of ischemic stroke and are associated with high rates of mortality and poor functional outcomes. Recent work exploring the molecular pathophysiology of the neurogliovascular unit in ischemic stroke suggests that deranged cellular ion homeostasis due to altered function and regulation of ion pumps, channels, and secondary active transporters plays an integral role in the development of cytotoxic and vasogenic edema and hemorrhagic conversion. Among these proteins involved in ion homeostasis, the ischemia-induced, nonselective cation conductance formed by the SUR1-TRPM4 protein complex appears to play a prominent role and is potently inhibited by glibenclamide, an FDA-approved drug commonly used in patients with Type 2 diabetes. Several robust preclinical studies have demonstrated the efficacy of glibenclamide blockade of SUR1-TRPM4 activity in reducing edema and hemorrhagic conversion in rodent models of ischemic stroke, prompting the study of the potential protective effects of glibenclamide in humans in an ongoing prospective phase II clinical trial. Preliminary data suggest glibenclamide significantly reduces cerebral edema and lowers the rate of hemorrhagic conversion following ischemic stroke, suggesting the potential use of glibenclamide to improve outcomes in humans.
\end{abstract}

(http://thejns.org/doi/abs/10.3171/2013.10.FOCUS13404)

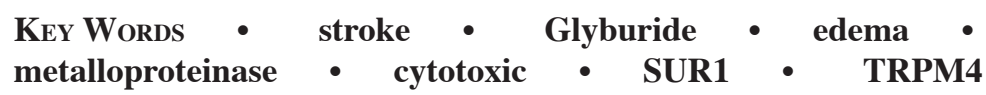

glibenclamide •

$\mathrm{C}$ EREBRAL edema and hemorrhagic conversion are major complications of ischemic stroke. Ischemic edema is the primary cause of death in patients with large infarctions: ${ }^{7}$ in major infarctions of the middle cerebral artery, the mortality of the "malignant edema syndrome" approaches $80 \% .^{1,27}$ Hemorrhagic conversion is associated with increased mortality and worse functional outcomes and occurs with a higher frequency in patients who receive thrombolytic therapy..$^{12}$ Recent insights into the pathophysiology of edema in stroke suggest that ischemic edema and hemorrhagic conversion share molecular antecedents and likely arise from similar processes of microvascular dysfunction..$^{20}$ Thus, there is

\footnotetext{
Abbreviations used in this paper: $\mathrm{ADC}=$ apparent diffusion coefficient; $\mathrm{BBB}=$ blood-brain barrier; $\mathrm{MCAO}=$ middle cerebral artery occlusion; $\mathrm{MMP}=$ matrix metalloproteinase $; \mathrm{mRS}=$ modified Rankin Scale; NIHSS = National Institutes of Health Stroke Scale; rt-PA $=$ recombinant tissue plasminogen activator; TRP $=$ transient receptor potential.
}

great interest in the development of therapies to preempt the occurrence of ischemic edema and hemorrhagic conversion by targeting the mechanisms of neurogliovascular dysfunction that underlie these phenomena.

A number of studies have implicated ionic flux through newly expressed SUR1-TRPM4 channels in the pathogenesis of edema and hemorrhagic conversion in focal ischemia. The SUR1-TRPM4 channel is a promising target, as it is potently and selectively blocked by the sulfonylurea glibenclamide (US trade name Glyburide), which has been safely used in the treatment of Type 2 diabetes mellitus for decades. Following a number of preclinical studies demonstrating the efficacy of this approach, trials on the use of glibenclamide in humans with ischemic stroke have been conducted or are currently underway. Preliminary data from these studies suggest that the blockade of SUR1-TRPM4 with glibenclamide may result in a significant reduction of ischemic edema, a lower incidence of hemorrhagic conversion, and improved outcomes. 
Here, we briefly review the pathogenesis of edema and hemorrhagic conversion in ischemic stroke as well as preclinical data indicating the role of SUR1-TRPM4 in this process. We then discuss the results both of studies examining the blockade of SUR1-TRPM4 in humans suffering from ischemic stroke and of an ongoing trial that may further elucidate the utility of glibenclamide in the prevention of ischemic edema and hemorrhagic conversion.

\section{Pathogenesis of Ischemic Edema and Hemorrhagic Conversion}

The pathogenesis of ischemic edema is thought to involve a stepwise progression through cytotoxic, ionic, and vasogenic edema, and ultimately hemorrhagic conversion. This stepwise progression is driven by pathological changes in the transmembrane permeability of neurons, glia, and vascular endothelial cells - the entire neurogliovascular unit. ${ }^{20,26}$ In the first phase, hypoxia-induced failure of energy-dependent mechanisms (primarily the $\mathrm{Na}^{+}-\mathrm{K}^{+}-$ adenosine triphosphatase [ATPase]) that maintain normal physiological ionic gradients across cell membranes causes thermodynamically driven abnormal ionic flux through channels and secondary active transporters. In particular, extracellular $\mathrm{Na}^{+}$flows down its concentration gradient into the intracellular compartment. This movement generates oncotic pressure that drives water into cells through aquaporins and other pathways, resulting in swelling and membrane blebbing of neurons, glia, and endothelial cells, features that characterize cytotoxic edema.

As ionic flux into cells depletes $\mathrm{Na}^{+}$from the extracellular space, an $\mathrm{Na}^{+}$gradient is established between the intravascular and extracellular spaces. This gradient drives transcapillary $\mathrm{Na}^{+}$movement across the bloodbrain barrier (BBB) through a host of preexisting and newly expressed channels and transporters. The $\mathrm{Na}^{+}$flux simultaneously provides electrochemical drive for $\mathrm{Cl}^{-}$ and oncotic drive for water to also flow into the extravascular space, resulting in an expansion of total extravascular brain volume, known as ionic edema.

During cytotoxic and ionic edema, swelling of endothelial cells causes cytoskeletal rearrangements, ${ }^{14}$ hypoxia activates a transcriptional program that increases endovascular permeability, and necrotic cell death continues in the ischemic core, which together contribute to further disruption of the BBB. These processes result in vasogenic edema, in which capillaries become fenestrated, tight junctions are disrupted, and reverse pinocytosis occurs, which together result in the leakage of macromolecules, ions, and water into the brain parenchyma.

Progressive breakdown of the BBB ultimately results in hemorrhagic conversion, the final phase of neurogliovascular dysfunction. Hemorrhagic conversion is marked by a complete failure of capillary integrity and extravasation of blood into the parenchyma and is associated with dramatically poorer outcomes. Progression to this stage occurs in about $10 \%$ of ischemic strokes, and the risk is worsened by the use of thrombolytic stroke therapy. ${ }^{6,10,11}$ Hemorrhagic conversion likely represents a continuation of the BBB disruption characteristic of vasogenic edema that is exacerbated by a number of factors, including oxidative stress, reperfusion injury, oncotic cell death, excitotoxicity, and increased extracellular proteolysis by matrix metalloproteinases (MMPs), ${ }^{5,24}$ among others. Matrix metalloproteinase activation is thought to be of particular clinical importance, as recombinant tissue plasminogen activator (rt-PA) has been shown to upregulate MMPs and probably contributes to the increased risk of hemorrhagic conversion in patients receiving rt-PA as thrombolytic therapy. ${ }^{25}$

Abnormal ionic flux through ion channels and secondary active transporters plays a central role in the development of the cytotoxic and ionic edema that ultimately results in vasogenic edema and hemorrhagic conversion. A number of such channels and transporters have been implicated in this process, including NKCC1, the $\mathrm{Na}^{+}-\mathrm{H}^{+}$ antiporter, acid-sensing ion channels, and transient receptor potential (TRP) channels. Of these, the monovalent cation channel formed by the association between SUR1 and TRPM4, herein referred to as SUR1-TRPM4, ${ }^{29}$ is an attractive target for therapeutic intervention, as it is inhibited by the widely used sulfonylurea antagonist glibenclamide, and preclinical studies suggest that it plays a major role in the development of ischemic edema.

\section{Role of SUR1-TRPM4 in Ischemic Edema and Hemorrhagic Conversion}

TRPM4 is a member of the TRPM subfamily of TRP channels that, under pathological conditions including ischemic stroke, associates with SUR1 to form SUR1TRPM4 channels. The channel conducts monovalent cations and is impermeable to divalent cations. Its opening requires nanomolar concentrations of intracellular $\mathrm{Ca}^{++}$, and it is opened when intracellular adenosine triphosphate (ATP) is depleted $(\mathrm{EC} 50 \cong 1 \mu \mathrm{M})$. The SUR1 subunit regulates the opening of SUR1-TRPM4 and is blocked with high affinity and specificity by the sulfonylurea antagonist glibenclamide $($ EC50 $=48 \mathrm{nM})$.

SUR1-TRPM4 is not constitutively expressed in the CNS; rather, its expression and subunit association is induced by hypoxia or injury. ${ }^{18}$ In rodent models of cerebral ischemia, levels of SUR1-encoding $A b c c 8$ mRNA increase 2.5 -fold at 3 hours after ischemic injury, and SUR1 protein expression is increased 2.5 -fold by 8 hours in the lesion and surrounding penumbra. ${ }^{18}$ This upregulation occurs in all cell types of the neurovascular unit (neurons, glia, and endothelial cells) and is not accompanied by upregulation of the inward rectifier potassium ion channel that is associated with SUR1 under normal conditions, indicating that the upregulated SUR1 becomes associated with TRPM4 in hypoxic injury.

A number of preclinical studies have shown that ionic movement through SUR1-TRPM4 is a major component of the pathological ionic flux in cytotoxic and ionic edema ${ }^{3}$ and that blockade of SUR1-TRPM4 with glibenclamide reduces edema, improves functional outcomes, and decreases mortality in rodent models of ischemic injury (Fig. 1). In a rat model of malignant cerebral edema caused by middle cerebral artery occlusion (MCAO), SUR1-TRPM4 blockade with glibenclamide reduces cerebral edema and 

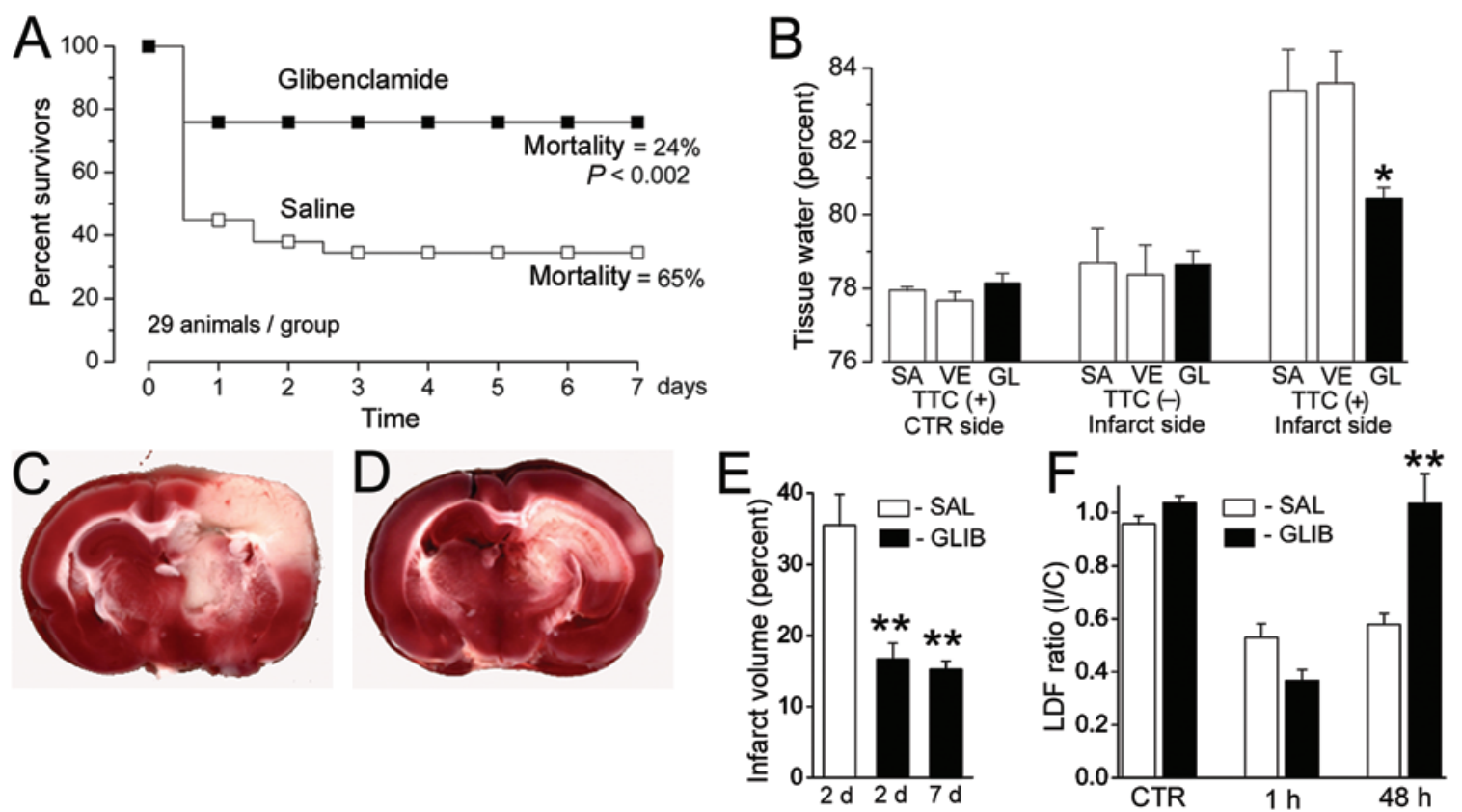

FIG. 1. SUR1-TRPM4 blockade with glibenclamide significantly reduces mortality, edema, and infarct volume and increases cerebral blood flow in a rodent model of ischemic stroke (MCAO). Mortality is significantly reduced $(p<0.002)$ following MCAO in rats treated with glibenclamide compared with controls (A). Bar graph showing the effect of glibenclamide (GL), compared with saline (SA) and vehicle DMSO (VE), on tissue water content after MCAO (B). Tissue in the ischemic core does not stain with 2,3,5-triphenyltetrazolium chloride (TTC-), but perilesional tissue does (TTC+). In perilesional tissue, treatment with glibenclamide significantly reduces tissue water content, consistent with its putative function of reducing cytotoxic edema by blockade of newly expressed SUR1-TRPM4, which is highly expressed in perilesional tissue. TTC-stained brain tissue section 2 days after MCAO in a rat treated with saline (C, infarct appears white). Compare with a TTC-stained section 2 days after MCAO in a rat treated with glibenclamide (D). Infarct volume is significantly reduced (E) at 2 and 7 days after MCAO by glibenclamide (GLIB), compared with saline (SAL). Laser Doppler flowmetry measurements of relative cerebral blood flow ratios between control and infarcted hemispheres (F). Forty-eight hours after MCAO, cerebral blood flow is significantly improved in rats treated with glibenclamide and has returned to preinfarct levels. Reprinted by permission from Macmillan Publishers Ltd: Nat Med, advance online publication, 19 March 2006 (doi: 10.1038/sj.nm.1390).

mortality by $50 \% .{ }^{18}$ Similar improvements in lesion volume and mortality are observed in thromboembolic, permanent MCAO, and transient MCAO with reperfusion rodent models of cerebral ischemia. ${ }^{23}$ The attenuation of infarct volume by glibenclamide is also associated with better functional outcomes. ${ }^{28}$ Treatment with glibenclamide, compared with decompressive craniectomy, results in superior outcomes in a rat model of malignant stroke. ${ }^{21} \mathrm{In}$ terestingly, glibenclamide may also decrease the risk of hemorrhagic conversion by reducing the expression, but not the enzymatic activity, of MMP-9, which is induced by ischemia. ${ }^{22}$ Because SUR1-TRMP4 is expressed de novo 3-8 hours after hypoxic injury, the treatment window of blockade with glibenclamide is quite long-edema is reduced when glibenclamide is administered up to 10 hours after ischemic injury. ${ }^{22}$

These preclinical studies strongly suggest that the blockade of SUR1-TRMP4 is a potential therapeutic strategy for reducing the pathological movement of ions that drives ischemic edema and hemorrhagic conversion. Glibenclamide is a potent inhibitor of the channel, has more than 10 times greater affinity for SUR1 than SUR2 (the latter with constitutive expression on cardiac, skeletal, and smooth muscle), and reduces SUR1-TRPM4-mediated cerebral edema at doses that are at least an order of magnitude below those associated with hypoglycemia in rodent models. Furthermore, it has a good safety profile that has been characterized over decades of use as therapy for Type 2 diabetes. For these reasons, there is strong interest in the use of SUR1-TRPM4 antagonists, particularly glibenclamide, as therapy to preempt the formation of edema following ischemic stroke in humans. Some retrospective analyses and preliminary results from ongoing clinical trials have indicated the validity of this approach.

\section{SUR1-TRPM4 Blockade in Humans With Ischemic Stroke}

A few retrospective analyses have compared stroke outcomes between patients with Type 2 diabetes taking a sulfonylurea drug for glycemic control and patients with Type 2 diabetes who are not taking a sulfonylurea to determine whether sulfonylureas are protective against ischemic edema and its associated sequelae. One study examined patients with Type 2 diabetes who were hospitalized within 24 hours of ischemic stroke. ${ }^{9}$ The authors identified 33 patients with diabetes who had continued to receive sulfonylureas throughout their admission and compared outcomes with those in a matched control cohort of 28 patients with diabetes who had not received sulfonylureas. The primary outcome was a decrease in 


\section{A. Khanna et al.}

the National Institutes of Health Stroke Scale (NIHSS) score of 4 or more or a reduction to 0 at discharge, and the secondary outcome was a modified Rankin Scale (mRS) score of 2 or less at discharge. Patients receiving sulfonylureas had significantly improved primary and secondary outcomes - the primary outcome was achieved in $36.4 \%$ of patients receiving a sulfonylurea versus $7.1 \%$ in the control group, and the secondary outcome was achieved in $81.8 \%$ versus $57.1 \%$, respectively. These differences were observed among patients with nonlacunar strokes and were independent of sex, history of ischemic attack, or blood glucose levels. A similar study was conducted in 2448 diabetic patients presenting with acute ischemic stroke to 11 stroke centers in Ontario. The study found that patients on sulfonylureas who had continued taking them during admission experienced significantly lower mortality than patients who had never taken sulfonylureas, even among those treated with rt-PA. ${ }^{17}$

Another retrospective study was conducted to determine whether sulfonylurea therapy conferred protection against symptomatic hemorrhagic transformation in patients with Type 2 diabetes. ${ }^{8}$ The authors compared 43 patients taking a sulfonylurea drug before and during hospital admission for ischemic stroke with 177 controls not taking a sulfonylurea drug. They found that the patients receiving sulfonylureas had significantly fewer deaths ( $0 \%$ vs $10 \%$ of controls) and a significantly lower rate of symptomatic hemorrhagic transformation ( $0 \%$ vs $11 \%$ of controls).

These retrospective studies were limited to patients with diabetes and so may have limited generalizability to nondiabetic patients. Even if the protective effects of sulfonylureas in stroke are limited to the diabetic patient population, these results are highly relevant, as approximately $33 \%$ of stroke patients are diabetic, and diabetic patients have poorer stroke outcomes. ${ }^{2}$ However, the hypothesized mechanism of protection by SUR1-TRPM4 blockade is theoretically applicable to nondiabetic patients. As such, prospective clinical trails of Glyburide for injection (RP-1127) as therapy for ischemic edema in nondiabetic patients are underway.

A phase I safety study confirmed the tolerability of intravenous RP-1127 administered as a bolus dose followed by a 3-day continuous infusion (NCT01132703, ClinicalTrials.gov). A subsequent multicenter, prospective, open-label, phase IIa trial, Glyburide Advantage in Malignant Edema and Stroke Pilot (GAMES-Pilot, NCT01268683, ClinicalTrials.gov), was conducted to demonstrate the feasibility of treating severe anterior circulation ischemic stroke with RP-1127 in patients with a baseline lesion volume between 82 and $210 \mathrm{~cm}^{3}$ on diffusion weighted imaging (that is, those in whom clinically significant edema was likely to occur) and able to begin infusion no more than 10 hours after symptom onset. Reports from this trial have suggested the efficacy of RP-1127 in reducing edema and the feasibility of further study (Fig. 2). Among 10 patients recruited for the trial, malignant edema occurred in only $20 \%$, compared with $88 \%$ in prospective observational studies of patients with comparable lesion volumes on diffusion weighted imaging. The mean increase in ipsilateral hemisphere volume
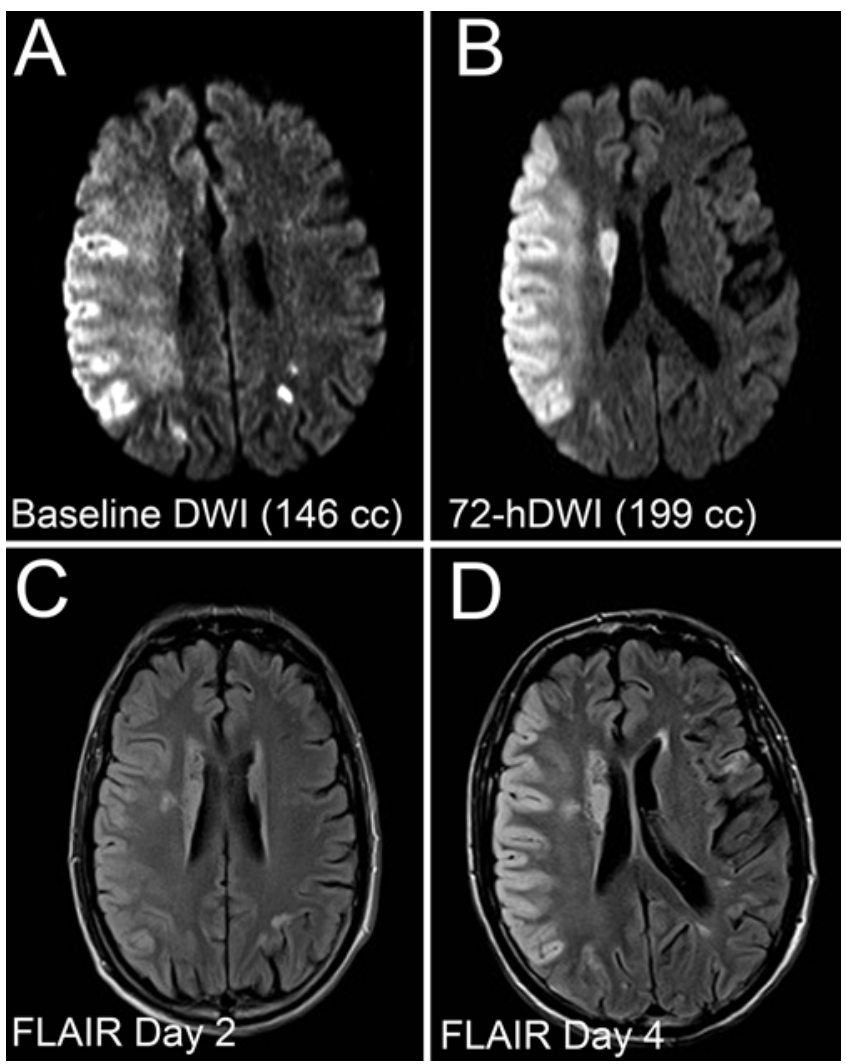

FIG. 2. Preliminary data from the GAMES-Pilot study indicate that glibenclamide (Glyburide) significantly reduces infarct size in humans with ischemic stroke. Diffusion weighted images (DWI) (A and B) and FLAIR images ( $C$ and $D$ ) obtained at indicated times after ischemia in a patient enrolled in the GAMES-Pilot trial who had presented with acute MCA syndrome, received conventional rt-PA, and developed an NIHSS score of 21. He received a 72-hour infusion of RP-1127 beginning approximately 9 hours after ischemia. The lesion volume expanded slightly by Day $3(\mathbf{B})$, but there was minimal mass effect. Note the unusual pattern of cytotoxic edema ( $C$ and $D$ ), which appears confined to the cortical gray matter, leaving the white matter intact. Gyral anatomy also appeared to be maintained. Reprinted by permission from Simard et al: Does inhibiting Sur1 complement rt-PA in cerebral ischemia? Ann N Y Acad Sci, John Wiley and Sons, 2012 New York Academy of Sciences.

was $50 \pm 33 \mathrm{~cm}^{3}$, compared with $71.5 \pm 27 \mathrm{~cm}^{3}$ in historical controls, and 9 of 10 patients had mRS scores of 4 or less after 30 days. ${ }^{15,16}$

Other reports from this trial give insight into the mechanism by which RP-1127 is hypothesized to exert its protective effects. To determine the utility of diffusion tensor imaging measurements as markers of cytotoxic edema in assessing response to RP-1127, diffusion tensor images for 9 patients from the GAMES-Pilot trial were compared with those a control cohort of 7 patients not treated with RP-1127. ${ }^{4}$ The ratio of the apparent diffusion coefficient (ADC) between tissues ipsilateral and contralateral to the lesion was significantly higher in patients treated with RP1127. In addition to indicating the utility of ADC measurements in evaluating the response to RP-1127, these results are consistent with the putative protective mechanism of $\mathrm{RP}-1127$, as high ADC ratios are thought to reflect a reduction in cytotoxic edema that would be expected from the SUR1-TRPM4 blockade. 
As described above, MMPs, particularly MMP-9, show increased expression and activity following ischemia. Upregulation and activity of MMP contribute to the risk of hemorrhagic conversion, and preclinical data suggest that glibenclamide can reduce the ischemic induction of MMP-9 activity by attenuating its expression. ${ }^{22}$ To determine whether this attenuation of expression occurs in humans, plasma samples from 6 subjects in the GAMESPilot were analyzed for levels of MMP-9 and compared with levels from a cohort of untreated patients. ${ }^{13}$ Average MMP-9 proenzyme levels among the Glyburide-treated patients were significantly lower than in controls (47.2 \pm $8.0 \mathrm{vs} 143.4 \pm 60.35 \mathrm{ng} / \mathrm{ml}$, respectively), and there was no difference in the endogenous MMP-9 inhibitor TIMP-1. Thus, RP-1127 may prevent hemorrhagic conversion in humans by inhibiting the expression of MMP-9 following ischemic injury, as it does in rodents. These findings also suggest that glibenclamide may complement rt-PA by counteracting the activation of MMPs by rt-PA, thereby mitigating the iatrogenic increase in the risk of hemorrhagic conversion when rt-PA is used as thrombolytic therapy. ${ }^{19}$

In light of these promising preliminary results, a multicenter, randomized, double-blind, phase II trial is underway to examine the efficacy of RP-1127 in the prevention of malignant edema in severe anterior circulation ischemic stroke (Glyburide Advantage in Malignant Edema and Stroke-Remedy Pharmaceuticals [GAMES-RP], NCT01794182, ClinicalTrials.gov). The primary outcome measures are the safety of RP-1127 and the proportion of patients with an mRS score no greater than 4 at Day 90 without the need for decompressive craniectomy. Secondary outcomes include the development of malignant edema, decompressive craniectomy, neurological deterioration, parenchymal hematomas, ipsilateral hemispheric swelling, 90-day mRS Score 0-3 and 0-4, Barthel Index at 90 days, and all-cause mortality at 90 days. Study completion in February 2015 is expected. There is hope that the results of the GAMES-RP trial and continued study into the efficacy of the glibenclamide blockade of SUR1TRPM4 may significantly improve outcomes following ischemic stroke.

\section{Disclosure}

This work was supported by grants from the National Heart, Lung and Blood Institute (Grant No. HL082517, J.M.S.) and the National Institute of Neurological Disorders and Stroke (Grant No. NS061808, J.M.S.); from the Brain Aneurysm Foundation (B.P.W.); and from the Manton Center for Orphan Diseases (K.T.K.). Dr. Simard holds a US patent (No. 7,285,574, Methods for treating neural cell swelling) and is a member of the scientific advisory board and holds shares in Remedy Pharmaceuticals. Remedy Pharmaceuticals provided no support for this project. All other authors report no conflict of interest concerning the materials or methods used in this study or the findings specified in this paper.

Author contributions to the study and manuscript preparation include the following. Conception and design: Simard, Walcott, Kahle. Acquisition of data: Khanna, Walcott. Analysis and interpretation of data: Simard, Khanna, Walcott. Drafting the article: Khanna, Walcott, Kahle. Critically revising the article: all authors. Reviewed submitted version of manuscript: all authors. Study supervision: Simard.

\section{References}

1. Berrouschot J, Sterker M, Bettin S, Köster J, Schneider D: Mortality of space-occupying ('malignant') middle cerebral artery infarction under conservative intensive care. Intensive Care Med 24:620-623, 1998

2. Callahan A, Amarenco P, Goldstein LB, Sillesen H, Messig M, Samsa GP, et al: Risk of stroke and cardiovascular events after ischemic stroke or transient ischemic attack in patients with type 2 diabetes or metabolic syndrome: secondary analysis of the Stroke Prevention by Aggressive Reduction in Cholesterol Levels (SPARCL) trial. Arch Neurol 68:1245-1251, 2011

3. Chen M, Simard JM: Cell swelling and a nonselective cation channel regulated by internal $\mathrm{Ca} 2+$ and ATP in native reactive astrocytes from adult rat brain. J Neurosci 21:6512-6521, 2001

4. Edlow BL, Wu O, Singhal AB, Chaudhry ZA, Yoo AJ, Stern $\mathrm{BJ}$, et al: Glyburide reduces cytotoxic edema within the cerebral peduncles in acute ischemic stroke. Stroke 44:ATP290, 2013 (Abstract)

5. Fujimura M, Gasche Y, Morita-Fujimura Y, Massengale J, Kawase M, Chan PH: Early appearance of activated matrix metalloproteinase-9 and blood-brain barrier disruption in mice after focal cerebral ischemia and reperfusion. Brain Res 842:92-100, 1999

6. Jaillard A, Cornu C, Durieux A, Moulin T, Boutitie F, Lees $\mathrm{KR}$, et al: Hemorrhagic transformation in acute ischemic stroke. The MAST-E study. Stroke 30:1326-1332, 1999

7. Kasner SE, Demchuk AM, Berrouschot J, Schmutzhard E, Harms L, Verro P, et al: Predictors of fatal brain edema in massive hemispheric ischemic stroke. Stroke 32:2117-2123, 2001

8. Kunte H, Busch MA, Trostdorf K, Vollnberg B, Harms L, Mehta RI, et al: Hemorrhagic transformation of ischemic stroke in diabetics on sulfonylureas. Ann Neurol 72:799-806, 2012

9. Kunte H, Schmidt S, Eliasziw M, del Zoppo GJ, Simard JM, Masuhr F, et al: Sulfonylureas improve outcome in patients with type 2 diabetes and acute ischemic stroke. Stroke 38:2526-2530, 2007

10. Larrue V, von Kummer R, del Zoppo G, Bluhmki E: Hemorrhagic transformation in acute ischemic stroke. Potential contributing factors in the European Cooperative Acute Stroke Study. Stroke 28:957-960, 1997

11. Lindley RI, Wardlaw JM, Sandercock PAG, Rimdusid P, Lewis SC, Signorini DF, et al: Frequency and risk factors for spontaneous hemorrhagic transformation of cerebral infarction. J Stroke Cerebrovasc Dis 13:235-246, 2004

12. Paciaroni M, Agnelli G, Corea F, Ageno W, Alberti A, Lanari A, et al: Early hemorrhagic transformation of brain infarction: rate, predictive factors, and influence on clinical outcome: results of a prospective multicenter study. Stroke 39:2249-2256, 2008

13. Pham L, O'Connor S, Yarbrough K, Jacobson S, Stern BJ, Sheth KN, et al: Intravenous glyburide treatment is associated with reduced matrix metalloproteinase- 9 in human acute stroke. Stroke 44:ATMP22, 2013 (Abstract)

14. Rajasekaran SA, Beyenbach KW, Rajasekaran AK: Interactions of tight junctions with membrane channels and transporters. Biochim Biophys Acta 1778:757-769, 2008

15. Sheth KN: Pilot GAMES (Glyburide Advantage in Malignant Edema and Stroke). Neurotherapeutics 10:545, 2013 (Abstract)

16. Sheth KN, Kimberly WT, Elm JJ, Kent TA, Mandava P, Yoo AJ, et al: Pilot study of intravenous glyburide in patients with a large ischemic stroke. Stroke [epub ahead of print], 2013

17. Silver FL, Fang J, Robertson AC, Casaubon L, Kapral MK: Possible neuroprotective effects of sufonylureas in diabetic patients with acute ischemic stroke. Stroke 40:e156, 2009 (Abstract)

18. Simard JM, Chen M, Tarasov KV, Bhatta S, Ivanova S, Mel- 


\section{A. Khanna et al.}

nitchenko L, et al: Newly expressed SUR1-regulated NC(CaATP) channel mediates cerebral edema after ischemic stroke. Nat Med 12:433-440, 2006

19. Simard JM, Geng Z, Silver FL, Sheth KN, Kimberly WT, Stern BJ, et al: Does inhibiting Surl complement rt-PA in cerebral ischemia? Ann N Y Acad Sci 1268:95-107, 2012

20. Simard JM, Kent TA, Chen M, Tarasov KV, Gerzanich V: Brain oedema in focal ischaemia: molecular pathophysiology and theoretical implications. Lancet Neurol 6:258-268, 2007

21. Simard JM, Tsymbalyuk N, Tsymbalyuk O, Ivanova S, Yurovsky V, Gerzanich V: Glibenclamide is superior to decompressive craniectomy in a rat model of malignant stroke. Stroke 41:531-537, 2010

22. Simard JM, Woo SK, Tsymbalyuk N, Voloshyn O, Yurovsky V, Ivanova S, et al: Glibenclamide-10-h Treatment Window in a Clinically Relevant Model of Stroke. Transl Stroke Res 3:286-295, 2012

23. Simard JM, Yurovsky V, Tsymbalyuk N, Melnichenko L, Ivanova S, Gerzanich V: Protective effect of delayed treatment with low-dose glibenclamide in three models of ischemic stroke. Stroke 40:604-609, 2009

24. Sumii T, Lo EH: Involvement of matrix metalloproteinase in thrombolysis-associated hemorrhagic transformation after embolic focal ischemia in rats. Stroke 33:831-836, 2002

25. Tsuji K, Aoki T, Tejima E, Arai K, Lee SR, Atochin DN, et al: Tissue plasminogen activator promotes matrix metallopro- teinase-9 upregulation after focal cerebral ischemia. Stroke 36:1954-1959, 2005

26. Walcott BP, Kahle KT, Simard JM: Novel treatment targets for cerebral edema. Neurotherapeutics 9:65-72, 2012

27. Walcott BP, Miller JC, Kwon CS, Sheth SA, Hiller M, Cronin $\mathrm{CA}$, et al: Outcomes in severe middle cerebral artery ischemic stroke. Neurocrit Care [epub ahead of print], 2013

28. Wali B, Ishrat T, Atif F, Hua F, Stein DG, Sayeed I: Glibenclamide administration attenuates infarct volume, hemispheric swelling, and functional impairments following permanent focal cerebral ischemia in rats. Stroke Res Treat 2012: 460909, 2012

29. Woo SK, Kwon MS, Ivanov A, Gerzanich V, Simard JM: The sulfonylurea receptor 1 (Surl)-transient receptor potential melastatin 4 (Trpm4) channel. J Biol Chem 288:3655-3667, 2013

Manuscript submitted September 13, 2013.

Accepted October 22, 2013.

Please include this information when citing this paper: DOI: 10.3171/2013.10.FOCUS13404.

Address correspondence to: J. Marc Simard, M.D., Ph.D., Department of Neurosurgery, 22 S. Greene St., Ste. S-12-D, Baltimore, MD 21201-1595.email: msimard@smail.umaryland.edu. 